gynecological malignancies, particularly after surgical procedures. Furthermore, we performed an economic analysis to provide an overview of HAIs-related costs.

Methodology We retrospectively collected data of the culture samples, collected in the microbiology laboratory, of patients recovered in the Oncological Gynecology Department of the Fondazione Policlinico "Agostino Gemelli" IRCCS, from 1st January 2017 to 31th December 2018. The data concerned both ordinary and emergency hospitalizations. For each patient, we collected data on germs responsible of infection and on the site of the infection

Result(s)* 323 gynecological cancer patients with HAIs were identified. 249 patients had undergone surgery in the previous 30 days and 74 were on chemotherapy treatment or in the follow-up phase. The most common HAIs were urinary infections $(57.9 \%)$, surgical wound infections were present in 42.1\%. $14.5 \%$ had central venous catheter infection and $21.7 \%$ of patients blood stream infections. The median length of stay for patients with post-operative infection or with an infective event during chemotherapy was 25 days. After discharge, $22 \%$ of patients were readmitted to the hospital due to new infection and were hospitalized for 22 days on average. The total cost that our hospital paid for the treatment of infected patients was 4.598391 \$.

Conclusion* Bowel resection appears to be the procedure most associated with the development of HAIs. An important method of reducing the risk of infections is careful screening of patients and risk factors before admission.

\section{PERIOPERATIVE ANAEMIA AND ITS PERILS}

S Patel ${ }^{*}$, S John, G Pactat, S Saso, S Thakrar. Queen Charlotte's and Chelsea Hospital, UK

\subsection{6/ijgc-2021-ESGO.311}

Introduction/Background* Anaemia (haemoglobin $<130 \mathrm{~g} / \mathrm{l}$ ) is an established, independent risk factor for perioperative morbidity and mortality ${ }^{1}$, the aetiology of which is diverse within in the gynaecological oncology population. We audited the management and consequences of perioperative anaemia in accordance with NICE guidelines ${ }^{2}$ in a quaternary gynaecological oncology unit in London, United Kingdom (UK).

Methodology We performed a retrospective electronic case note review of women undergoing major (M) or major-plus (MP) gynaecological oncology surgeries between $1^{\text {st }}$ September 2019 and $29^{\text {th }}$ February 2020. The category of operation was standardised at the unit.

Result(s)* Of 236 notes audited, 58.5\% ( $\mathrm{n}=138)$ were anaemic pre-operatively.

$87 / 236$ patients underwent $M$ surgery and 149/236 patients underwent MP surgery. The prevalence of anaemia was $43.7 \%$ in the $M$ cohort $(n=38)$ versus $67.1 \%$ in the MP cohort ( $=100)$. Mean $\mathrm{Hb}$ was the same between groups $(113.4 \mathrm{~g} / \mathrm{L}$ vs. $113.4 \mathrm{~g} / \mathrm{L})$. Only $4 \%(\mathrm{n}=6)$ of patients had iron studies sent pre-operatively. No patients received an iron infusion preoperatively, whilst $2.9 \%$ of patients had pre-operative blood transfusions.

The mean estimated blood loss (EBL) for all groups was $289.4 \mathrm{ml}$. $53 \%$ of patients $(\mathrm{n}=125)$ did not have any documented operative EBL. The overall incidence of post-operative blood transfusion was $24.6 \%(n=58)$. Women identified with pre-operative anaemia had a higher incidence of post-operative blood transfusion (anaemic 35.5\% vs. non anaemic 9.2\%).
Post-operative transfusion incidence

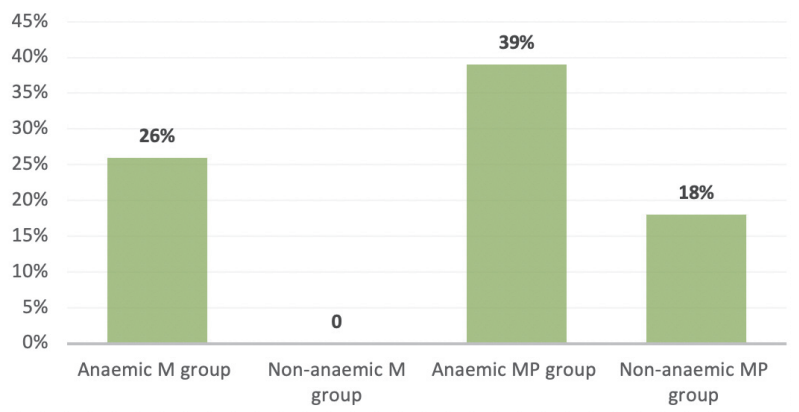

Abstract 651 Figure 1 Post-operative transfusion incidence

Mean length of stay (LOS) was greater in anaemic M (3.4 vs.2.2 days) and MP (6.9 days vs. 5.1 days) procedures compared to their non-anaemic counterparts.

Conclusion* Our analysis reveals a need for improvement in the management of peri-operative anaemia in our population. It also demonstrates the peri-operative consequences of untreated anaemia including a higher incidence of post-operative blood transfusion and increased LOS.

Our department is working to define a specific anaemia management pathway for all patients undergoing $\mathrm{M}$ or $\mathrm{MP}$ gynaecological surgery and we aim to undertake a randomised control trial to show our results.

- Musallam KM et al. Preoperative anaemia and postoperative outcomes in non-cardiac surgery: a retrospective cohort study Lancet. 2011 Oct 15;378(9800):1396-407. doi: 10.1016/S0140-6736(11)61381-0. Epub 2011 Oct 5.

National Institute for Health and Care Excellence. Perioperative care in adults; NICE guideline 2020 (Clinical guideline [NG180]). Available from https://www.nice.org.uk/guidance/ ng180

\section{AN AUDIT AND FEEDBACK INTERVENTION TO MONITOR QUALITY OF CARE OF OVARIAN CANCER ACCORDING TO ESGO GUIDELINES IN THE PIEMONTE CANCER NETWORK}

${ }^{1} \mathrm{~A}$ Ferrero*, ${ }^{2}$ E Pagano, ${ }^{3} \mathrm{M}$ Mistrangelo, ${ }^{1} \mathrm{~L}$ Fuso, ${ }^{2} \mathrm{VH}$ Martins, ${ }^{4} \mathrm{G}$ Valabrega, ${ }^{5} \mathrm{ME}$ Laudani, ${ }^{4} \mathrm{~F}$ Marocco, ${ }^{6} \mathrm{D}$ Surico, ${ }^{7} \mathrm{E}$ Piovano, ${ }^{8} \mathrm{M}$ Barbero, ${ }^{9} \mathrm{M}$ Camanni, ${ }^{10} \mathrm{EM}$ Delpiano, ${ }^{11} \mathrm{~A}$ Puppo, ${ }^{11} \mathrm{~A}$ Daniele, ${ }^{12} \mathrm{~L}$ Zavallone, ${ }^{13} \mathrm{~V}$ Aguggia, ${ }^{14} \mathrm{R}$ Fiorentino, ${ }^{2} \mathrm{G}$ Ciccone, ${ }^{5} \mathrm{P}$ Zola. ${ }^{1}$ Mauriziano Hospital, Academic Department Gynaecology and Obstetrics, Torino, Italy; ${ }^{2}$ Città della Salute e della Scienza, University of Turin, Department of Epidemiology, Torino, Italy; ${ }^{3}$ Città della Salute e della Scienza, Department of Piemonte and Valle d'Aosta Cancer Network, Torino, Italy; ${ }^{4}$ Candiolo Cancer Institute, FPO-IRCCS, Department of Oncology, Candiolo, Italy; ${ }^{5}$ Città della Salute e della Scienza, University of Turin, Gynaecological Oncology Unit, Department Surgical Sciences, Torino, Italy; ${ }^{6}$ University of Eastern Piedmont, Obstetrics and Gynecology Clinic, Novara, Italy; 'Regina Montis Regalis Hospital, Obstetrics and Gynecology Unit, Mondovi, Italy; ${ }^{8}$ Cardinal Massaia Hospital, Obstetrics and Gynaecology, Asti, Italy; ${ }^{9}$ Gradenigo Hospital, Gynecological Surgery, Torino, Italy; ${ }^{10}$ Martini Hospital, Obstetrics and Gynaecology, Torino, Italy; ${ }^{11}$ Santa Croce e Carle Hospital, Obstetrics and Gynaecology, Cuneo, Italy; ${ }^{12}$ Infermi Hospital, Department of Medical Oncology, Ponderano, Biella, Italy; ${ }^{13}$ SS. Antonio e Biagio Hospital, Obstetrics and Gynaecology, Alessandria, Italy; ${ }^{14}$ Castelli Hospital, Obstetrics and Gynaecology, Verbania, Italy

\subsection{6/ijgc-2021-ESGO.312}

Introduction/Background* Epithelial ovarian cancer (EOC) is the most lethal gynaecological cancer with 3285 estimated deaths in Italy in 2021. In 2016, the Piedmont and Valle d'Aosta Oncology Network (NW Italy) started on an Audit 